\title{
SOX2 promotes the migration and invasion of laryngeal cancer cells by induction of MMP-2 via the PI3K/Akt/mTOR pathway
}

\author{
NING YANG, LIAN HUI, YAN WANG, HUIJUN YANG and XUEJUN JIANG \\ Department of Otorhinolaryngology, The First Affiliated Hospital of \\ China Medical University, Shenyang 110001, P.R. China
}

Received February 14, 2014; Accepted March 12, 2014

DOI: $10.3892 /$ or.2014.3120

\begin{abstract}
SOX2 is a high mobility group box containing transcription factor that has been reported to be aberrantly overexpressed in various human malignancies, including laryngeal squamous cell carcinoma (LSCC). However, the potential role of SOX2 in LSCC migration and invasion remains to be elucidated. In the present study, we generated stable transformants of human LSCC cells constitutively overexpressing SOX 2 and investigated the effects of SOX 2 overexpression on migration and invasion in LSCC cells as well as the possible underlying mechanisms. We found that ectopic overexpression of SOX2 in LSCC cells enhanced their migratory and invasive ability in vitro, accompanied by increased expression and activity of matrix metalloproteinase (MMP)-2. Meanwhile, SOX2-induced cell migration and invasion were significantly abrogated by a neutralizing anti-MMP-2 antibody or small interfering RNA targeting MMP-2. Furthermore, overexpression of SOX2 induced phosphorylation of Akt and mammalian target of rapamycin (mTOR), which are downstream effectors of the PI3K pathway. Finally, LY294002, an inhibitor of PI3K, also markedly abolished SOX2-induced activation of the Akt/mTOR pathway and increased cell invasion and MMP-2 expression. Taken together, we conclude that SOX2 promotes migration and invasion of laryngeal cancer cells by inducing MMP-2 via the PI3K/Akt/mTOR pathway. Our findings suggest that SOX 2 may serve as a potential therapeutic target for LSCC.
\end{abstract}

\section{Introduction}

Laryngeal squamous cell carcinoma (LSCC), which represents the vast majority $(\sim 96 \%)$ of laryngeal carcinoma cases, is one

Correspondence to: Dr Xuejun Jiang, Department of Otorhinolaryngology, The First Affiliated Hospital of China Medical University, 155 North Nanjing Street, Shenyang 110001, P.R. China E-mail: cmulhjiangxuejun@163.com

Key words: SOX2, laryngeal cancer, cell invasion, matrix metalloproteinase-2, PI3K of the most common malignancies of the head and neck and accounts for $1.5 \%$ of all types of cancers (1). In accordance with the American Cancer Society data, LSCC caused $0.7 \%$ of cancer-related death in 2009 and its incidence is increasing over time (2). Early-stage LSCC is often cured by surgery or radiotherapy, while the prognosis of patients with advanced LSCC remains poor with a low 5-year survival rate. Due to tumor recurrence and metastasis, the clinical outcome has not shown satisfactory improvement in the last three decades despite advances in surgical management, radiotherapy and chemotherapy (3). Therefore, understanding the molecular mechanisms involved in the metastatic process is imperative to identify novel potential targets for developing more effective therapeutic strategies against LSCC.

SOX2, a member of the SRY-related high mobility group box (SOX) family of transcription factors (4), plays an essential role in the maintenance of pluripotency and self-renewal of embryonic stem cells, and generation of induced pluripotent stem cells (5-7). Recent evidence indicates that SOX2 is also involved in tumorigenesis (8). Twenty-eight percent of all breast carcinomas and $43 \%$ of basal-like triple-negative breast cancers present with overexpression of SOX2 (9). SOX2 genes were also found to be amplified in a subset of squamous cell lung and esophageal cancers (10). In addition, SOX2 overexpression was found in glioblastoma (11), non-small cell lung $(12,13)$, prostate (14), pancreatic (15) and ovarian cancer (16), and hepatocellular carcinoma (17), suggesting an oncogenic role of SOX2. In particular, recently, Tang et al reported that SOX2 is frequently overexpressed in LSCC, and overexpression of SOX 2 is associated with unfavorable patient prognosis (18). To the best of our knowledge, however, the functional role of SOX2 in the initiation and progression of LSCC remains to be elucidated.

In the present study, we established a stable LSCC cell line constitutively overexpressing SOX2 and investigated the effects of SOX2 overexpression on LSCC cell invasion and migration as well as the possible underlying mechanisms.

\section{Materials and methods}

Cell culture. Human LSCC Hep-2 cells were obtained from the Cell Bank of the Chinese Academy of Sciences (Shanghai, 
China) and were cultured in a 1:1 mixture of Ham's F12 and Dulbecco's modified Eagle's medium (DMEM) with $10 \%$ fetal bovine serum (FBS), $100 \mathrm{U}$ of penicillin and $100 \mathrm{mg} / \mathrm{ml}$ streptomycin. The cells were maintained at $37^{\circ} \mathrm{C}$ in a humidified atmosphere containing $5 \% \mathrm{CO}_{2}$.

Generation of plasmid constructs and establishment of SOX2-overexpressing cell lines. To generate the SOX2 overexpression vectors, the coding sequence of the SOX 2 gene was obtained by RT-PCR and cloned into the pEGFP-N1 vector. Hep-2 cells were transfected with the pEGFP-N1-SOX2 vector to induce excessive SOX2 expression or the pEGFP-N1 vector to generate stable clones expressing SOX1 constitutively as control. Two days after transfection, G418 solution was added to the cells for selection of the stable clones. Stable clones were selected and maintained in medium containing G418. The resulting cell lines were designated as Hep-2/pEGFPN1-SOX2 and Hep-2/pEGFP-N1, respectively.

Quantitative RT-PCR ( $q R T-P C R)$. Total RNA was extract from cells using RNA Simple Total RNA kit (Tiangen Co., Beijing, China), and $3 \mu \mathrm{g}$ of RNA was converted into cDNA using the High-Capacity cDNA Archive kit (Applied Biosystems, Foster City, CA, USA). The primers were synthesized based on published sequences $(19,20)$. For detection of SOX2 mRNA the following primers were used: forward, 5'-AACCAAGACGCTCATGAAGAAG-3' and reverse, 5'-CT GCGAGTAGGACAT GCTGTAG-3'. For detection of MMP-2 mRNA the following primers were used: forward, 5'-TGG CAAFTCGGCTTCTGTC-3' and reverse, 5'-TTCTTGTCGCG GTCGTAGTC-3'. For each PCR reaction, a master mix that included SYBR-Green Master Mix (Solarbio Co., Beijing, China), the forward primer, the reverse primer and $10 \mathrm{ng}$ template cDNA was prepared. The PCR conditions consisted of $5 \mathrm{~min}$ at $95^{\circ} \mathrm{C}$ followed by 40 cycles of $95^{\circ} \mathrm{C}$ for $30 \mathrm{sec}, 60^{\circ} \mathrm{C}$ for $30 \mathrm{sec}$ and $72^{\circ} \mathrm{C}$ for $30 \mathrm{sec}$. Data were analyzed using the comparative $\Delta \mathrm{Ct}$ method using GAPDH as an internal normalization control.

Western blot analysis. Western blot analysis was performed using a standard protocol. The primary antibodies used in the present study were as follows: rabbit anti-SOX 2 (1:2,000; Wanlei Life Science Co., Ltd., Shenyang, China), rabbit anti-MMP-2, anti-PCNA, anti-cyclin D1, anti-p-Akt, anti-Akt, anti-pmTOR and anti-mTOR (1:2,000; Santa Cruz Biotechnology, Santa Cruz, CA, USA). The secondary antibodies used in this study were alkaline phosphatase peroxidase-conjugated antirabbit IgG. Detection was performed using the BCIP/NBT Alkaline Phosphatase Color Development kit (Beyotime Institute of Biotechnology, Shanghai, China) according to the manufacturer's instructions.

MTT assay. Untransfected Hep-2 cell lines and the stably transfected lines were replated onto 96-well plates at $1 \times 10^{5}$ cells/well and cultured overnight to allow for cell attachment. At daily intervals (24, 48, 72 and $96 \mathrm{~h})$, the number of viable cells was determined by 3-[4,5-dimethylthiazol-2-yl]2,5-diphenyltetrazolium bromide (MTT) assay as previously described (21). Briefly, MTT solution $(5 \mathrm{mg} / \mathrm{ml}$ ) was added to each well at indicated times, and the formazan precipitate was dissolved in dimethyl sulfoxide $(100 \mu \mathrm{l})$ after $2 \mathrm{~h}$ of incubation. For colorimetric analysis, the absorbance at $492 \mathrm{~nm}$ was recorded using an ELISA reader (Thermo Molecular Devices Co., Union City, CA, USA).

Colony formation assay. The detailed protocol was as described previously (22). Briefly, cells were seeded in dish at a density of 1,000 cell $/ \mathrm{ml} / \mathrm{dish}$, and colony formation was allowed to proceed for 14 days at $37^{\circ} \mathrm{C}$ in a humidified atmosphere with $5 \% \mathrm{CO}_{2}$. Cells were then fixed with formaldehyde, and individual colonies were stained with Wright-Giemsa. After washing and drying, the colony counts were conducted using an inverted microscope (XDS-1B; Beijing, China). Aggregates of 50 or more cells were considered as one colony.

Wound scratch assay. Each well of a 24-well tissue culture plate was seeded with cells to a final density of 100,000 cells/well and these cells were maintained at $37^{\circ} \mathrm{C}$ and $5 \% \mathrm{CO}_{2}$ for $24 \mathrm{~h}$ to permit cell adhesion and the formation of a confluent monolayer. These confluent monolayers were then scored with a sterile pipette tip to leave a scratch of $\sim 0.4-0.5 \mathrm{~mm}$ in width. The cell surface was then washed with serum-free culture medium for three times to remove dislodged cells. Wound closure was monitored by collecting digitized images at 0,12 and $24 \mathrm{~h}$ after the scratch was performed. Digitized images were captured with an inverted microscope (Motic China Group Co., Xiamen, China) and digital camera (Nikon, Tokyo, Japan). The digitized images were then analyzed using ImageJ software as previously described (23).

Invasion assay. Transwells (24-well) coated with Matrigel (8- $\mu \mathrm{m}$ pore size; BD Biosciences, San Jose, CA, USA) were used for cell invasion assays (24). Equal numbers $\left(1 \times 10^{5}\right)$ of cells were plated onto a separate well. Cells were starved overnight in serum-free medium, trypsinized and washed three times in DMEM containing $1 \%$ FBS. A total of $1 \times 10^{5}$ cells were then resuspended in $500 \mu \mathrm{l}$ DMEM containing $1 \% \mathrm{FBS}$ and added to the upper chamber, while MEM with $10 \%$ FBS was added to the lower chamber as a chemoattractant. For the control, medium containing 1\% FBS was added to the lower chamber. After $24 \mathrm{~h}$ of incubation, the Matrigel and the cells remaining in the upper chamber were removed by cotton swabs. The cells on the lower surface of the membrane were fixed in formaldehyde and stained with hematoxylin staining solution. The cells in at least five random microscopic fields (magnification, x200) were counted and photographed.

Gelatin zymography. Gelatin zymography was performed using a Genius kit (Genetic Medicine Technology Company, Shanghai, China) according to the manufacturer's instructions. In short, the liquid level was adjusted according to the number of cells. MMP-2 standards (Sigma, St. Louis, MO, USA) were added to the substrate as a reference. Electrophoresis was performed until the dye reached the bottom of the gel. The gel was then taken to be treated with refolding solution, digestion solution and dye solution. Next, bleaching solution was used to treat the gel until a clear white band appeared against a dark blue background. The band area and grayscale values were analyzed using a gel image analysis system. 
A

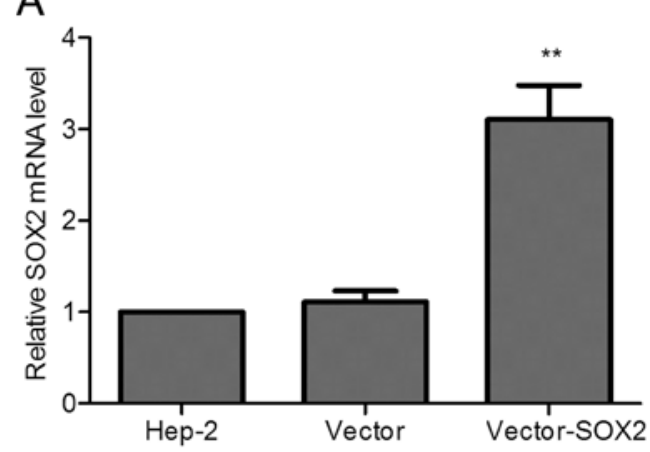

B
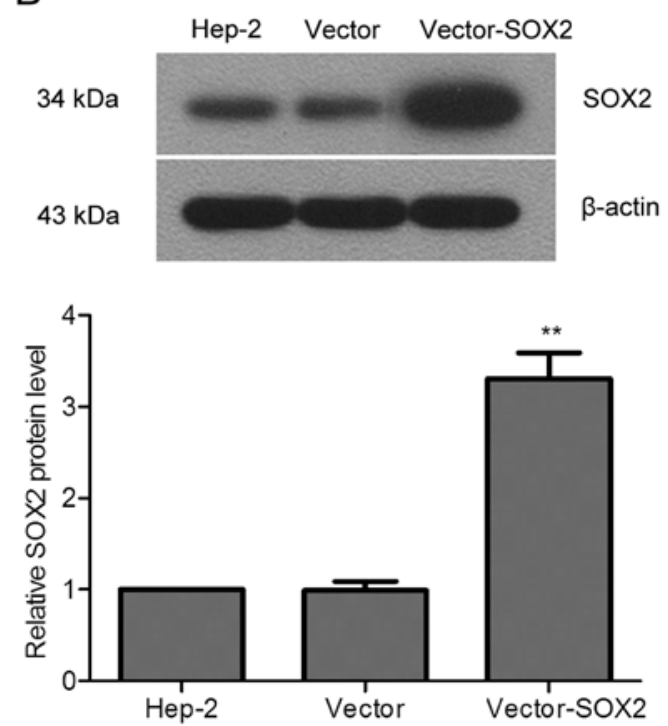

Figure 1. Overexpression of SOX2 in Hep-2 cells was confirmed by qRT-PCR and western blot analysis. (A) SOX2 mRNA level was measured by qRT-PCR. Data are shown as means \pm SD of triplicate experiments. (B) SOX2 protein level was detected by western blot analysis. Densitometric quantification data are expressed as SOX2 relevant to $\beta$-actin. ${ }^{* *} \mathrm{P}<0.01$ compared with the empty vector-transfected cells.

Transfection of MMP-2 siRNA. Sense and antisense oligonucleotides targeting MMP-2 were synthesized according to previously published sequences (25). Non-specific siRNA which has no target in the human transcriptome was used as a negative control. siRNA transfection of cells was performed as previously described (25). Briefly, 1 day before transfection cells were plated at $5 \times 10^{3}$ cells/well in a 96 -well culture plate and allowed to reach $30 \%$ confluency after $24 \mathrm{~h}$ of incubation. The transfection mixture containing MMP-2-targeted siRNA and Lipofectamine 2000 (Invitrogen, Carlsbad, CA, USA) was incubated for $20 \mathrm{~min}$ at room temperature before being applied to the cells. The cells were then incubated for $6 \mathrm{~h}$ at $37^{\circ} \mathrm{C}$ in a humidified atmosphere containing $5 \% \mathrm{CO}_{2}$. Subsequently, cells were washed with PBS and maintained in culture medium for $48 \mathrm{~h}$ before expression of MMP-2 was assessed with western blot analysis.

Statistical analysis. Data presented in the present study are means \pm standard error of the mean. Statistical analysis of differences between groups was performed using analysis of variance (ANOVA) followed by Dunnett's t-test. The statis- tical significance of the differences between mean values was defined as $\mathrm{P}<0.05$.

\section{Results}

Overexpression of SOX2 promotes cell proliferation in Hep-2 cells. To confirm whether the established cell line could stably express high levels of SOX2, the expression levels of SOX2 mRNA and protein were determined by qRT-PCR and western blot analysis, respectively. Our results showed that Hep-2/pEGFP-N1-SOX2 cells stably expressed a significantly higher level of SOX2 when compared with these levels in the Hep-2/pEGFP-N1 or parental Hep-2 cells (Fig. 1A and 1B). Next, we examined the effects of SOX2 overexpression on Hep-2 cell proliferation by MTT assay. SOX2-overexpressing cells showed a time-dependent increase in cell proliferation, which was significantly higher than controls (Fig. 2A). Overexpression of SOX2 in Hep-2 cells also promoted anchorage-independent growth, as demonstrated by colony formation assay (Fig. 2B). Moreover, the expression level of PCNA, an essential protein involved in cell proliferation, was also examined. As shown in Fig. 2C, Hep-2 cells transfected with the SOX2 gene had significantly higher levels of PCNA, supporting the regulatory role of SOX2 in Hep-2 cell growth.

Overexpression of SOX2 leads to an increase in Hep-2 cell migration and invasion. To determine whether the alteration of SOX2 expression leads to changes in cell migration and invasion, wound scratch and Transwell assays were performed, respectively. SOX2-overexpressing clones (Hep-2/ pEGFP-N1-SOX2) showed a $>2$-fold increase in the cell migration rate when compared to the rate in the vector control and parental Hep-2 cells at 12 and $24 \mathrm{~h}$, respectively (Fig. 3A). In vitro invasion ability was determined by the number of cells that migrated across the membrane after a 24-h incubation. As shown in Fig. 3B, a significant increase in cell invasion was observed in the SOX2-overexpressing Hep-2 cells. These abovementioned results suggest that SOX2 overexpression significantly enhanced cell migration and invasion in vitro in the Hep-2 cells.

Altered SOX2 expression affects cell migration and invasion by regulating MMP-2 in Hep-2 cells. MMP-2 is a factor which has been proven to function in tumor metastasis, thus the expression and activity of MMP-2 were examined in the present study to further determine the mechanism by which overexpression of SOX2 affects migration and invasion. The mRNA and protein levels of MMP-2 in the stably transfected cells were determined by qRT-PCR and western blot analysis, respectively. We found that Hep-2 cells with SOX2 overexpression showed high levels of MMP-2 expression when compared with the level of expression in the controls (Fig. 4A and 4B). In addition, MMP-2 activity was significant higher in the Hep-2/ pEGFP-N1-SOX2 cells when compared with the activity in the controls (Fig. 4C). These above results indicate that SOX2 overexpression causes increased expression and activity of MMP-2 in Hep-2 cells. To investigate whether SOX2 overexpression promotes cell migration and invasion via regulation of MMP-2, the MMP-2 gene was silenced in Hep-2/pEGFPN1-SOX2 cells using MMP-2-targeted siRNA. Western blot 

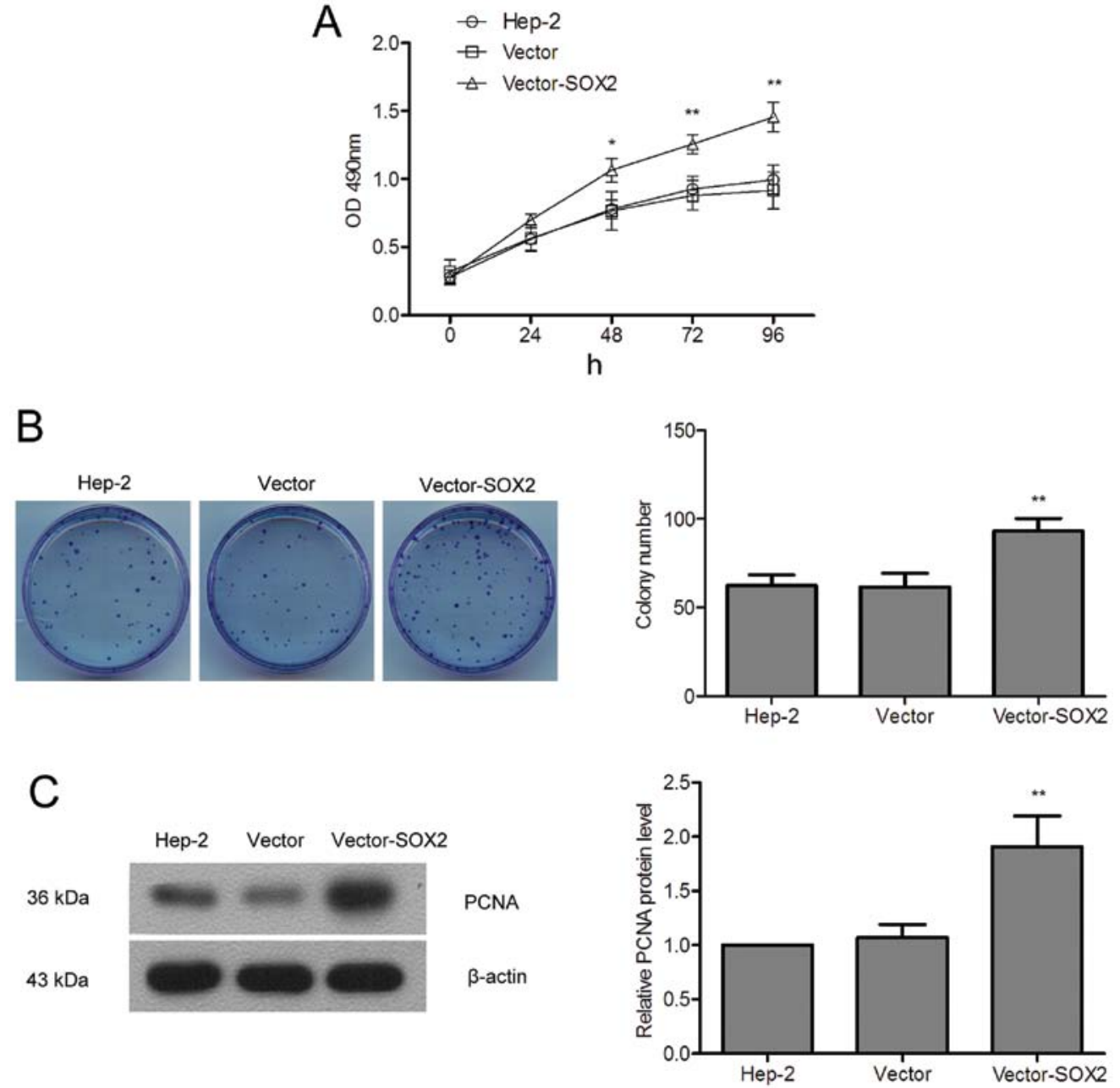

Figure 2. Overexpression of SOX2 promotes Hep-2 cell proliferation. (A) Cell viability was measured by MTT assay at the indicated times. Data are shown as means \pm SD of triplicate experiments. (B) Anchorage-independent cell growth was evaluated by colony formation assay. Representative colony formation results are shown. (C) PCNA protein level was detected by western blot analysis. Densitometric data of three separate experiments are shown. ${ }^{*} \mathrm{P}<0.05,{ }^{* *} \mathrm{P}<0.01$ compared with the empty vector-transfected cells.

analysis confirmed that MMP-2 expression was effectively suppressed by $>70 \%$ compared with the cells transfected with control siRNA or Hep-2/pEGFP-N1-SOX2 cells (Fig. 5A). Next, the effects of MMP-2 knockdown on cell migration and invasion were examined. As shown in Fig. 5B, cell migration of Hep-2/pEGFP-N1-SOX2 cells was significantly reduced after transfection with MMP-2-targeted siRNA, compared with the migration rate in the Hep-2/pEGFP-N1-SOX2 cells transfected with control siRNA $(\mathrm{P}<0.01)$, whereas no significant difference was found between control siRNA-transfected and parental Hep-2/pEGFP-N1-SOX2 cells. Cell invasion assay also demonstrated that suppression of MMP-2 expression in Hep-2/pEGFP-N1-SOX2 cells resulted in a significant decrease in cell invasion compared with the control siRNA-transfected and parental Hep-2/pEGFP-N1-SOX2 cells (Fig. 5C). To further demonstrate the role of MMP-2 in cell migration and invasion induced by SOX2 overexpression, Hep-2/pEGFP-N1-SOX2 cells were treated with an anti-MMP-2 antibody with irrelevant IgG as control. Compared with the untreated Hep-2/pEGFPN1-SOX2 cells, cells treated with anti-MMP-2 antibodies presented significantly lower migration (Fig. 5D) and invasive capabilities (Fig. 5E). Collectively, these results indicate that the regulatory effect of SOX 2 on Hep-2 cell migration and invasion was, at least in part, mediated by MMP-2.

SOX2 regulates MMP-2 expression through the PI3K/Akt/ mTOR signaling pathway. It has been reported that the $\mathrm{PI} 3 \mathrm{~K} / \mathrm{Akt}$ pathway is associated with tumor progression and P13K/Akt-mediated cell signals induce the expression of MMPs including MMP-2 (26). Therefore, we examined the effects of SOX 2 on activation of the PI3K/Akt signaling pathway to explore whether Akt functions as an essential kinase in MMP-2 expression induced by SOX2 overexpression. As shown in Fig. 6A, the expression levels of phosphorylated Akt and phosphorylated mTOR were markedly increased in SOX2overexpressing cells compared with the controls. However, LY294002, a selective PI3K inhibitor, effectively abolished the increased expression levels of MMP-2 and phosphorylated Akt and mTOR (Fig. 6B). Meanwhile, the Transwell assay also showed that cell invasion was significantly reduced in the LY294002-treated Hep-2/pEGFP-N1-SOX2 cells (Fig. 6C). Collectively, these results demonstrated that activation of the PI3K/Akt signaling pathway is involved in the SOX2-induced MMP-2-mediated modulation of cell invasion. 

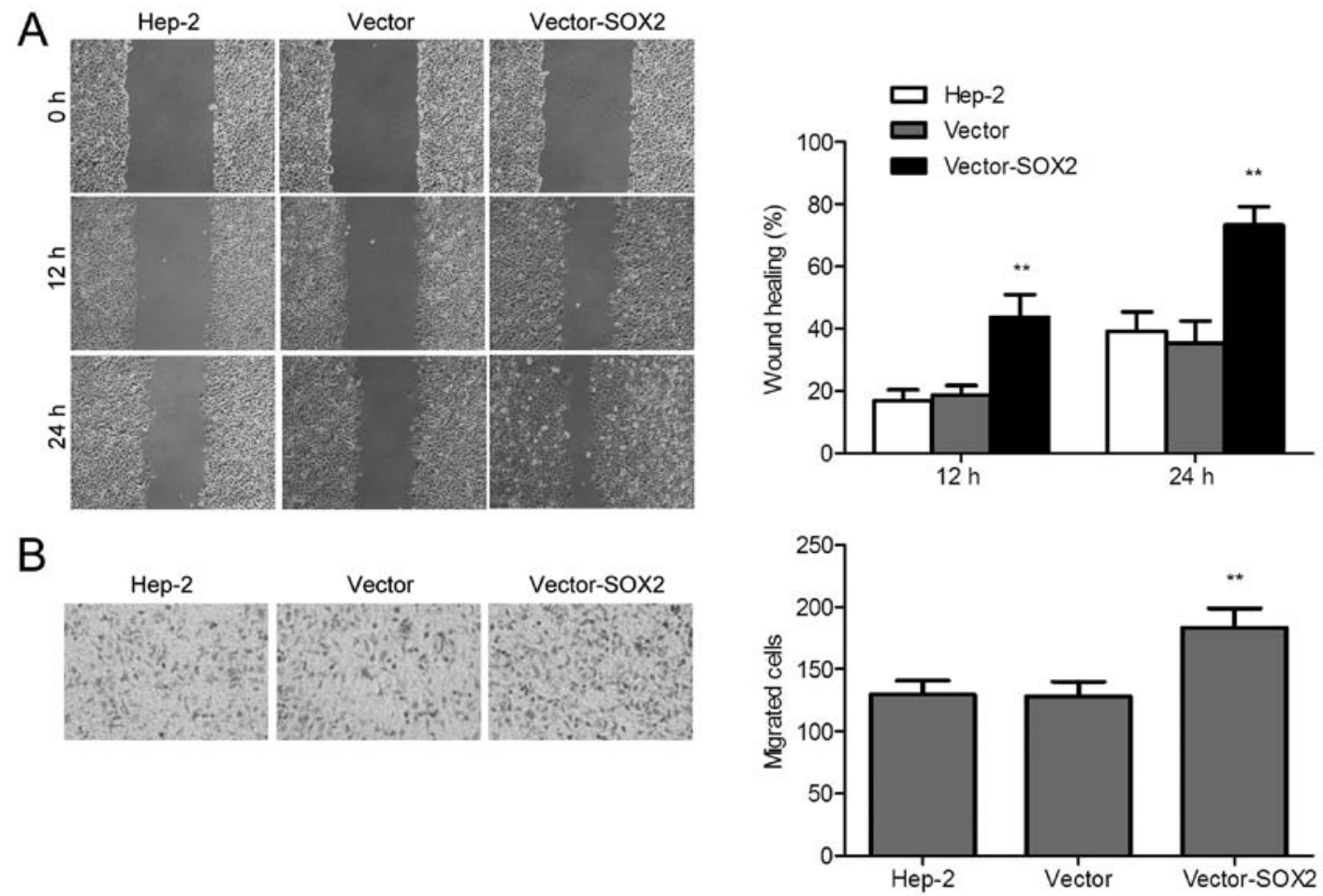

Figure 3. SOX2 overexpression enhances Hep-2 cell migration and invasion in vitro. (A) Cell migration was detected by wound scratch assay. Representative wound closure images from three experiments are shown. Cell migration was calculated and expressed as the percentage of cell coverage to the initial cell-free zone. Values are presented as means \pm SD of three independent experiments. (B) Cell invasion was determined by Transwell assay. Representative images of the invaded cells (stained with hematoxylin) selected from three experiments are shown. Migrated cells were counted under a microscope. ${ }^{* *} \mathrm{P}<0.01$ compared with the empty vector-transfected cells.
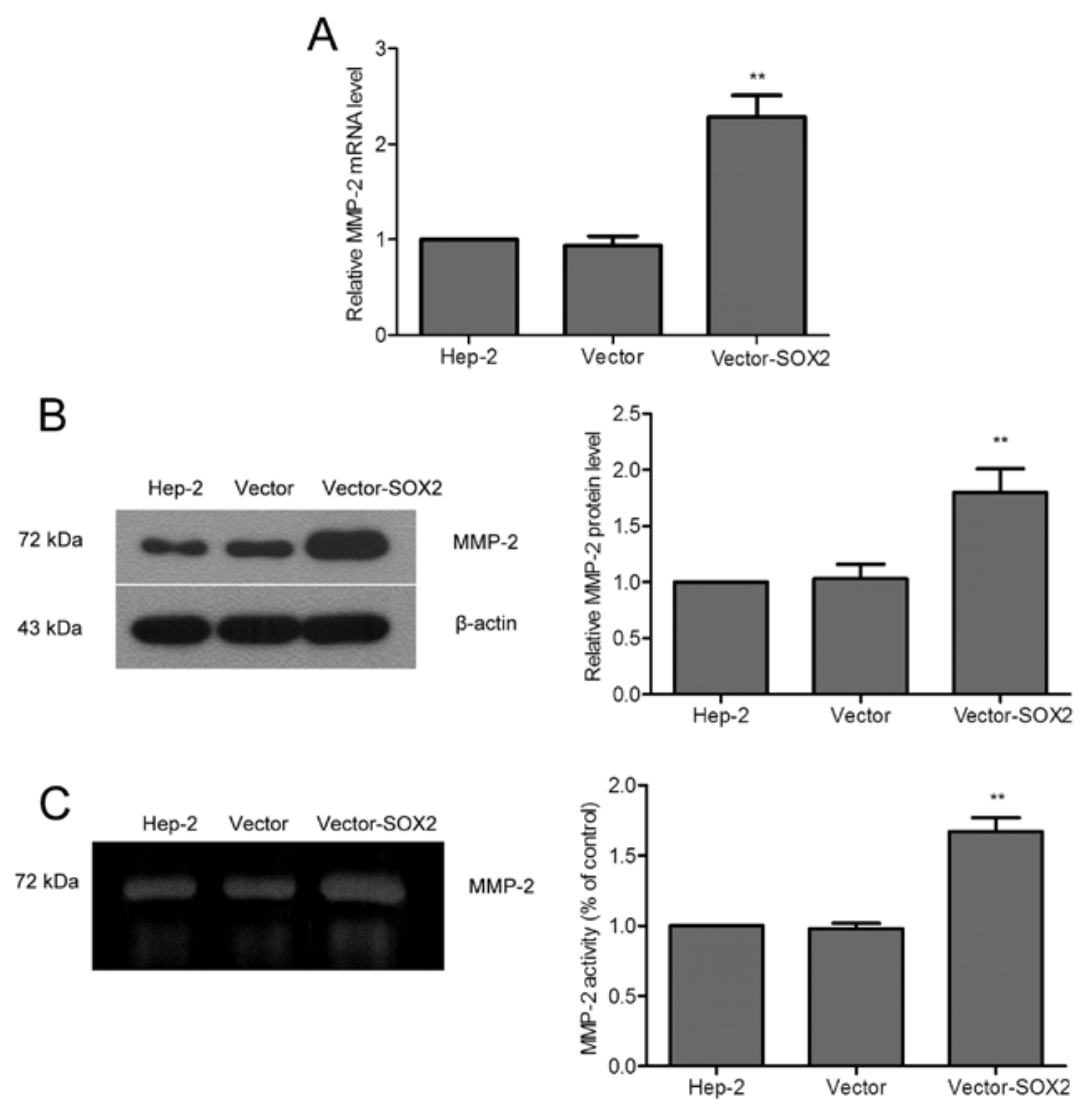

Figure 4. Overexpression of SOX2 induces an increase in MMP-2 expression and activity. (A) The mRNA level of MMP-2 was measured by qRT-PCR. Data are shown as means \pm SD of triplicate experiments. (B) The protein level of MMP-2 was detected by western blot analysis. Quantitative analysis was performed by densitometry. (C) MMP-2 activity was analyzed by gelatin zymography. The bands were analyzed by densitometry and data are shown as means \pm SD of triplicate experiments. ${ }^{* *} \mathrm{P}<0.01$ compared with the empty vector-transfected cells. MMP-2, matrix metalloproteinase- 2 . 
A

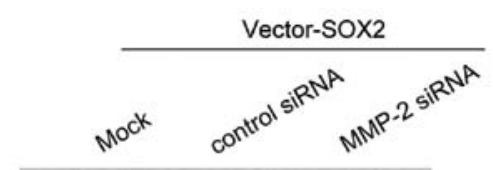

$72 \mathrm{kDa}$

$43 \mathrm{kDa}$

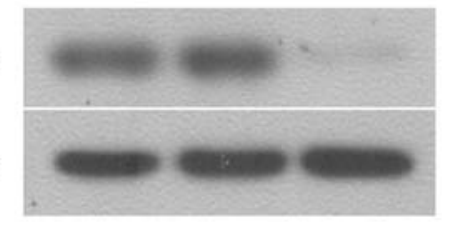

MMP-2

$\beta$-actin

\section{B}

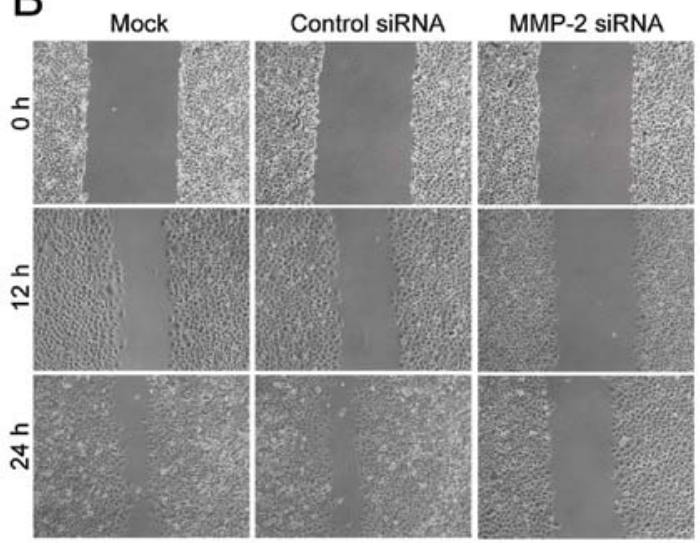

C
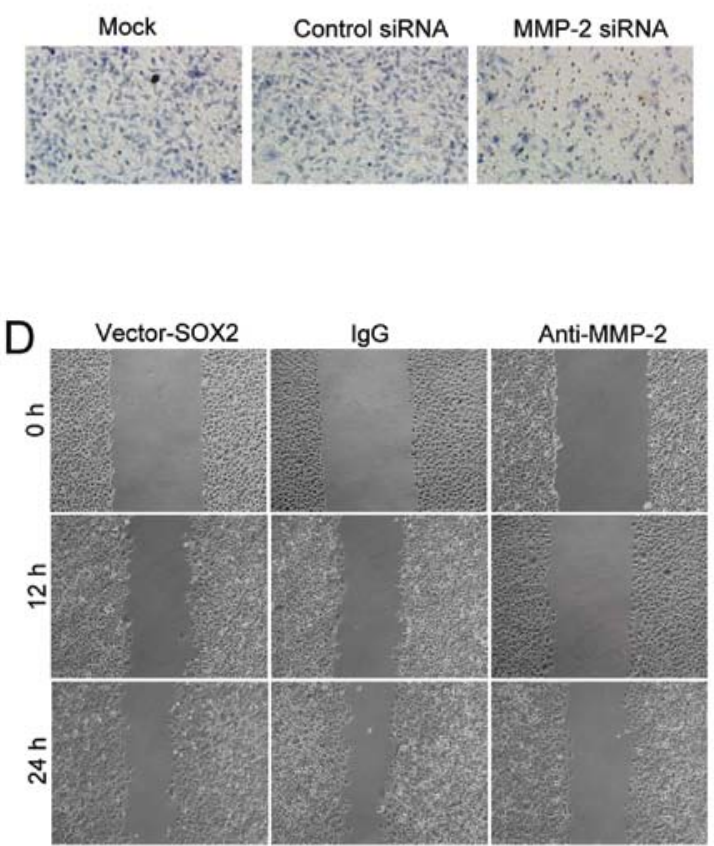

$\mathrm{E}$

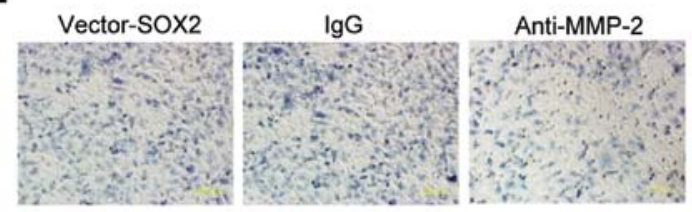

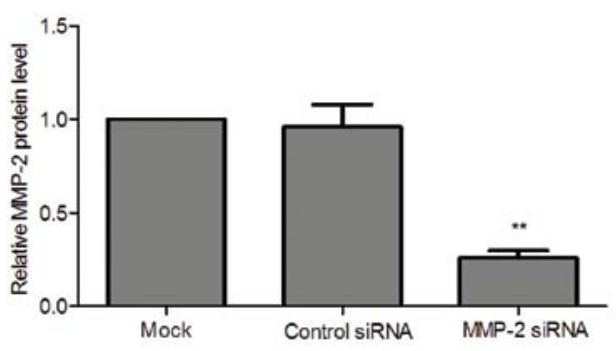
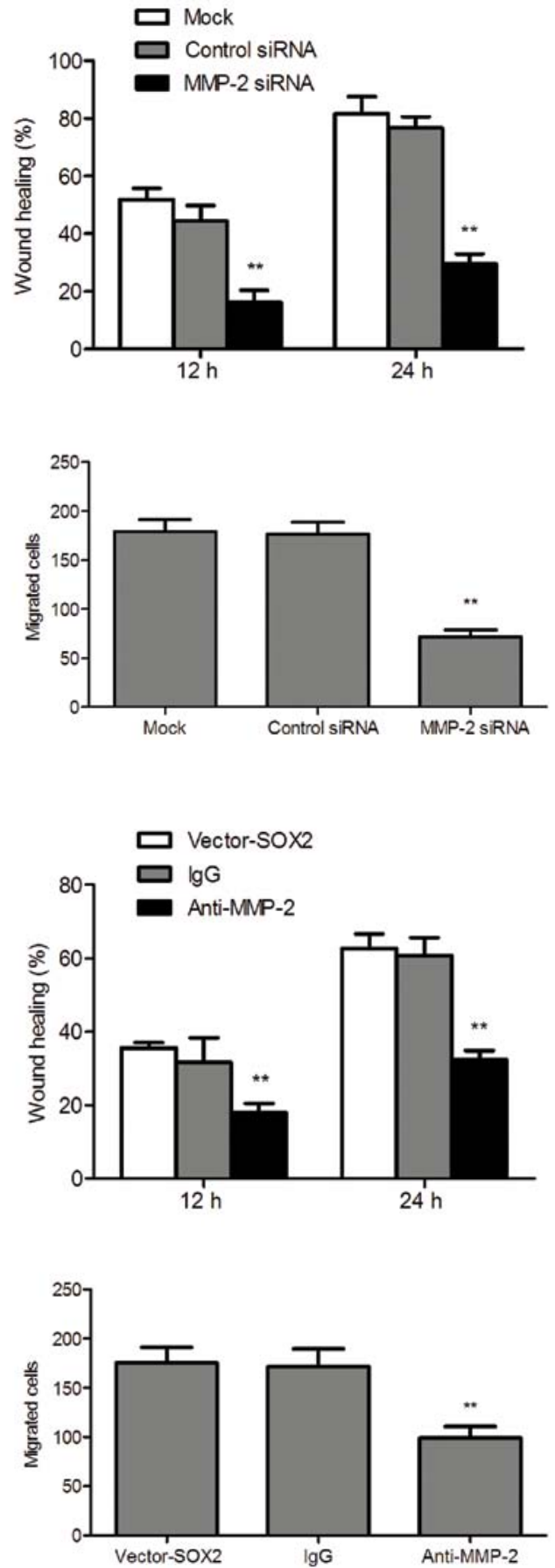

Figure 5. Overexpression of SOX2 promotes cell migration and invasion by increasing MMP-2 expression. (A) siRNA-mediated suppression of MMP-2 was confirmed by western blot analysis. Scramble siRNA was used as the control. (B and C) The effects of MMP-2 knockdown on cell migration and invasion in Hep-2/pEGFP-N1-SOX2 cells were evaluated by (B) wound scratch and (C) Transwell assays, respectively. ${ }^{* *}$ P $<0.01$ compared with control siRNA. (D and E) The effects of an anti-MMP-2-antibody on cell migration and invasion in Hep-2/pEGFP-N1-SOX2 cells were evaluated by (D) wound scratch and (E) Transwell assays, respectively. Irreverent IgG was used as the control. ${ }^{* *} \mathrm{P}<0.01$ compared with IgG. MMP-2, matrix metalloproteinase-2. 

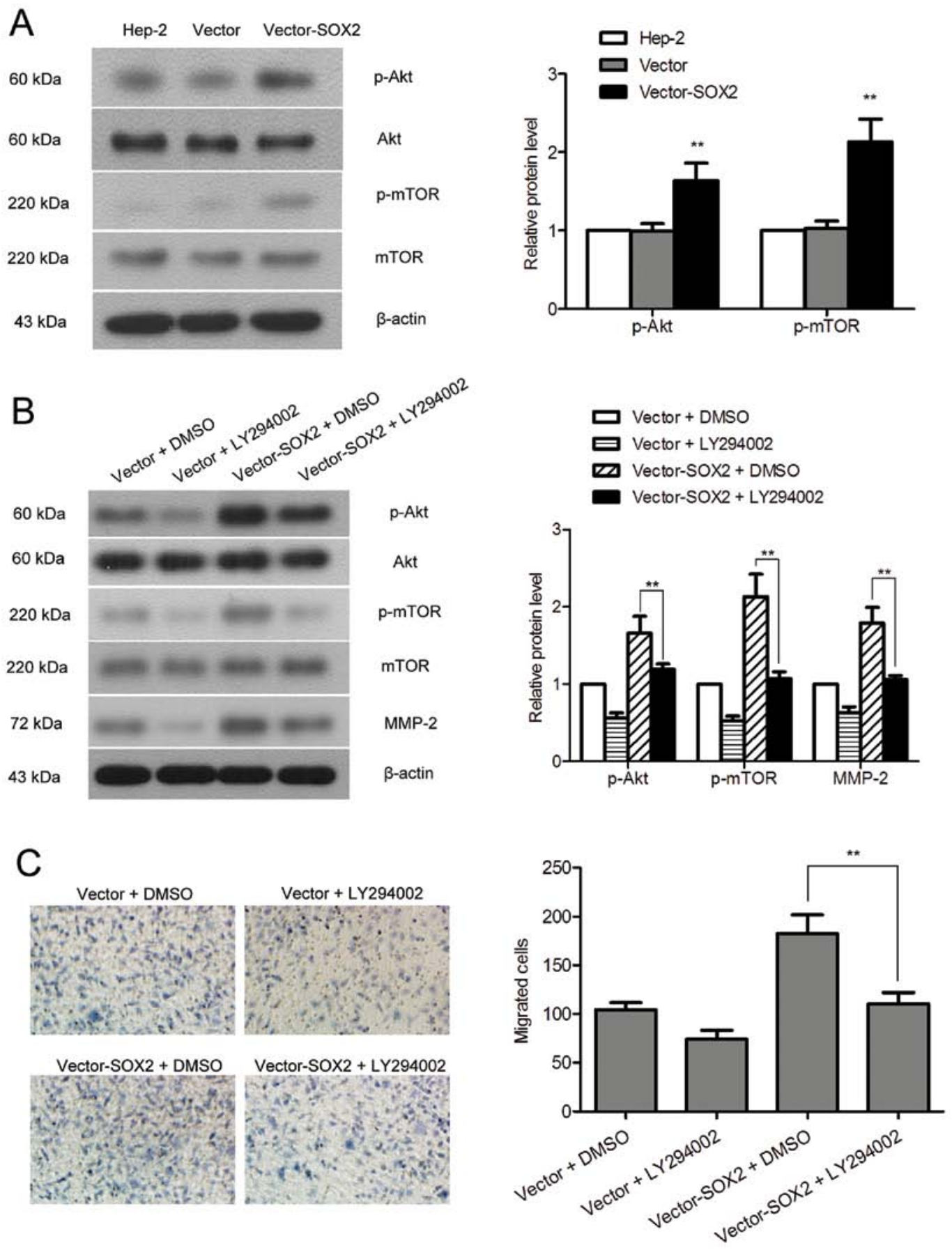

Figure 6. SOX2 enhances the invasion of Hep-2 cells by inducing MMP-2 through the PI3K/Akt/mTOR pathway. (A) The expression of Akt, mTOR, phosphorylated Akt and phosphorylated mTOR was detected by western blot analysis. (B) The effects of PI3K inhibition by the specific inhibitor LY294002 on MMP-2 expression were determined by western blot analysis. Cells were incubated with $20 \mu \mathrm{M}$ LY294002 for 30 min and then harvested for western blot analysis. DMSO was used as control. Data are shown as means \pm SD of triplicate experiments. (C) The effects of LY294002 on cell invasion in Hep-2/pEGFPN1-SOX2 cells were evaluated by Transwell assay. MMP-2, matrix metalloproteinase-2.

\section{Discussion}

SOX2, a self-renewal transcription factor crucial to maintain pluripotency in embryonic stem cells (ESCs), has been reported to play an important role in the development of numerous malignancies (6). Ben-Porath et al proposed that SOX2 is expressed in a subpopulation of cells within the tumor with tumor-initiating characteristics (27). A more recent study found that this subpopulation of cells present remarkable similarities in their overall gene expression profile with stem cells and exhibit crucial phenotypic characteristics, including sustained proliferation and resistance to apoptotic signals (28). Therefore, targeting the SOX2 gene may offer a unique chance for anticancer intervention by interfering with tumor initiation and progression.

A recent study indicated that SOX2 overexpression is correlated with poor prognosis in LSCC and may contribute to the malignant progression of LSCC (18). Thus, elucidation of the cellular function of SOX2 to determine how it contributes to LSCC is important. In the present study, we 
investigated the role of SOX2 in LSCC progression and invasion, and characterized the signaling pathway by which SOX2 affects the biological processes of tumor cells by overexpressing the SOX2 gene in LSCC cell lines. We found overexpression of SOX2 in LSCC Hep-2 cells promoted cell proliferation, migration and invasion. The present study also provides mechanistic insight into how SOX2, via activation of PI3K/Akt/mTOR pathway, regulates these above cellular functions by demonstrating that (i) SOX2 overexpression is associated with P13K/Akt/mTOR activation in Hep-2 cells; (ii) SOX2 overexpression induces an increase in cell invasion induced in a PI3K-dependent manner and (iii) Akt plays a part in mediating the effect of SOX2 on cell invasion, at least in part, by regulating MMP-2 expression.

Previous studies have demonstrated that SOX2 promotes the proliferation of cervical cancer and breast cancer cells $(29,30)$. Consistent with these findings, the present study showed that SOX 2 overexpression led to a significant higher time-dependent cell proliferation in Hep-2 cells when compared with the controls. We also examined the level of PCNA, an important biomarker of cell proliferation, to further confirm the pro-proliferative effect of SOX2 overexpression. Western blot analysis revealed a much higher level of PCNA in Hep-2/pEGFP-N1-SOX2 cells than the level in the controls, suggesting the role of SOX2 as an oncogene in promoting Hep-2 cell proliferation.

A highly invasive nature is one of the characteristics of LSCC (18). Therefore, we investigated the effect of SOX2 overexpression on Hep-2 cell migration and invasion. Our results showed that an increase in SOX2 expression significantly enhanced the migration and invasion of Hep-2 cells. Next, we examined MMP-2 expression to explore the correlation between SOX 2 expression and cell migration and invasion. MMPs, a family of zinc-dependent endopeptidases, are required in extracellular matrix (ECM) degradation associated with cancer cell invasion, metastasis and angiogenesis. In particular, the role of MMP-2, which is a type IV collagenase, in tumor cell migration and invasion during carcinogenesis is well documented (31). In the present study, elevated expression of SOX2 correlated well with an increase in MMP-2 levels, indicating a functional interaction between SOX2 and MMP-2. This possible relationship was further investigated using MMP-2-targted siRNA and an anti-MMP-2 antibody. Our results demonstrated that transfection with MMP-2targeted siRNA or treatment with the anti-MMP-2 antibody significantly inhibited the cell migration and invasion induced by SOX 2 overexpression, suggesting that SOX 2 induces cell migration and invasion in an MMP-2-dependent manner.

Results from epidemiological studies and animal studies have shown that P13K induces the activation of MMP-2, MMP-9 and urokinase-type plasminogen activator (uPA), resulting in ECM degradation (32). Previous studies also reported that PI3K activation is crucial for MMP production in tumor cell lines (33). However, it remains unknown as to whether SOX2 overexpression is associated with activation of the P13K/Akt pathway, and activation of PI3K/Akt acts as an upstream signal in SOX2-induced MMP-2 expression in Hep-2 cells. Furthermore, we found that SOX2 induced MMP-2 expression in a PI3K-dependent manner. More importantly, the experimental data demonstrated that SOX2-induced
PI3K/Akt pathway played a crucial role in Hep-2 cell invasion, and blocking of P13K signaling by a specific inhibitor almost completely abrogated the SOX2 overexpression-induced increase in cell invasion. Taken together, these data suggest that the invasion-promoting effect of SOX2 in Hep-2 cells was mediated by a cellular signaling pathway involving PI3K/Akt and MMP-2.

In summary, our findings in the present study indicate that SOX2 upregulation in Hep-2 cells promotes cell proliferation, enhances cell invasiveness by increasing MMP-2 expression and activity. Furthermore, our results suggest that SOX2 induces these significant changes in cell invasion via activation of the PI3K/Akt/mTOR signaling pathway. Although more studies are needed, the present study suggests that SOX2 may be a potential molecular target for developing novel gene targeted therapy against LSCC.

\section{Acknowledgements}

This study was supported by a grant from the Natural Science Foundation of Liaoning Province (no. 201202287).

\section{References}

1. Shah JP, Karnell LH, Hoffman HT, et al: Patterns of care for cancer of the larynx in the United States. Arch Otolaryngol Head Neck Surg 123: 475-483, 1997.

2. Jemal A, Siegel R, Ward E, Hao Y, Xu J and Thun MJ: Cancer statistics, 2009. CA Cancer J Clin 59: 225-249, 2009.

3. Zhao XD, Zhang W, Liang HJ and Ji WY: Overexpression of miR-155 promotes proliferation and invasion of human laryngeal squamous cell carcinoma via targeting SOCS1 and STAT3. PLoS One 8: e56395, 2013.

4. Pevny LH and Lovell-Badge R: Sox genes find their feet. Curr Opin Genet Dev 7: 338-344, 1997.

5. Masui S, Nakatake Y, Toyooka Y, et al: Pluripotency governed by Sox 2 via regulation of $O c t 3 / 4$ expression in mouse embryonic stem cells. Nat Cell Biol 9: 625-635, 2007.

6. Fong H, Hohenstein KA and Donovan PJ: Regulation of selfrenewal and pluripotency by Sox 2 in human embryonic stem cells. Stem Cells 26: 1931-1938, 2008.

7. Takahashi K, Tanabe K, Ohnuki M, et al: Induction of pluripotent stem cells from adult human fibroblasts by defined factors. Cell 131: 861-872, 2007.

8. Hussenet T and du Manoir S: SOX2 in squamous cell carcinoma: amplifying a pleiotropic oncogene along carcinogenesis. Cell Cycle 9: 1480-1486, 2010.

9. Rodriguez-Pinilla SM, Sarrio D, Moreno-Bueno G, et al: Sox2: a possible driver of the basal-like phenotype in sporadic breast cancer. Mod Pathol 20: 474-481, 2007.

10. Wilbertz T, Wagner P, Petersen K, et al: SOX2 gene amplification and protein overexpression are associated with better outcome in squamous cell lung cancer. Mod Pathol 24: 944-953, 2011.

11. Gangemi RM, Griffero F, Marubbi D, et al: $S O X 2$ silencing in glioblastoma tumor-initiating cells causes stop of proliferation and loss of tumorigenicity. Stem Cells 27: 40-48, 2009.

12. Lu Y, Futtner C, Rock JR, et al: Evidence that SOX2 overexpression is oncogenic in the lung. PLoS One 5: e11022, 2010.

13. Tung CL, Hou PH, Kao YL, et al: SOX2 modulates alternative splicing in transitional cell carcinoma. Biochem Biophys Res Commun 393: 420-425, 2010.

14. Bae KM, Su Z, Frye C, et al: Expression of pluripotent stem cell reprogramming factors by prostate tumor initiating cells. J Urol 183: 2045-2053, 2010.

15. Sanada Y, Yoshida K, Ohara M, Oeda M, Konishi K and Tsutani Y: Histopathologic evaluation of stepwise progression of pancreatic carcinoma with immunohistochemical analysis of gastric epithelial transcription factor SOX2: comparison of expression patterns between invasive components and cancerous or nonneoplastic intraductal components. Pancreas 32: 164-170, 2006. 
16. Ye F, Li Y, Hu Y, Zhou C, Hu Y and Chen $\mathrm{H}$ : Expression of Sox2 in human ovarian epithelial carcinoma. J Cancer Res Clin Oncol 137: 131-137, 2011

17. $\mathrm{Xu} \mathrm{XL}$, Xing BC, Han HB, et al: The properties of tumorinitiating cells from a hepatocellular carcinoma patient's primary and recurrent tumor. Carcinogenesis 31: 167-174, 2010.

18. Tang XB, Shen XH, Li L, Zhang YF and Chen GQ: SOX2 overexpression correlates with poor prognosis in laryngeal squamous cell carcinoma. Auris Nasus Larynx 40: 481-486, 2013.

19. Adya R, Tan BK, Punn A, Chen J and Randeva HS: Visfatin induces human endothelial VEGF and MMP-2/9 production via MAPK and PI3K/Akt signalling pathways: novel insights into visfatin-induced angiogenesis. Cardiovasc Res 78: 356-365, 2008.

20. Stolzenburg S, Rots MG, Beltran AS, et al: Targeted silencing of the oncogenic transcription factor SOX2 in breast cancer. Nucleic Acids Res 40: 6725-6740, 2012.

21. Wang Y, Zhao Y, Liu Y, Tian L and Jin D: Chamaejasmine inactivates Akt to trigger apoptosis in human HEp-2 larynx carcinoma cells. Molecules 16: 8152-8164, 2011.

22. Qin FX, Shao HY, Chen XC, et al: Knockdown of NPMI by RNA interference inhibits cells proliferation and induces apoptosis in leukemic cell line. Int J Med Sci 8: 287-294, 2011.

23. Walter MN, Wright KT, Fuller HR, MacNeil S and Johnson WE: Mesenchymal stem cell-conditioned medium accelerates skin wound healing: an in vitro study of fibroblast and keratinocyte scratch assays. Exp Cell Res 316: 1271-1281, 2010.

24. Liao W, Liu W, Yuan Q, et al: Silencing of DLGAP5 by siRNA significantly inhibits the proliferation and invasion of hepatocellular carcinoma cells. PLoS One 8: e80789, 2013.

25. Ries C, Egea V, Karow M, Kolb H, Jochum $M$ and Neth $P$ : MMP-2, MT1-MMP, and TIMP-2 are essential for the invasive capacity of human mesenchymal stem cells: differential regulation by inflammatory cytokines. Blood 109: 4055-4063, 2007.
26. Chen X, Liao J, Lu Y, Duan X and Sun W: Activation of the PI3K/Akt pathway mediates bone morphogenetic protein 2 -induced invasion of pancreatic cancer cells Panc-1. Pathol Oncol Res 17: 257-261, 2011.

27. Ben-Porath I, Thomson MW, Carey VJ, et al: An embryonic stem cell-like gene expression signature in poorly differentiated aggressive human tumors. Nat Genet 40: 499-507, 2008.

28. Schoenhals M, Kassambara A, De Vos J, Hose D, Moreaux J and Klein B: Embryonic stem cell markers expression in cancers. Biochem Biophys Res Commun 383: 157-162, 2009.

29. Ji J and Zheng PS: Expression of Sox 2 in human cervical carcinogenesis. Hum Pathol 41: 1438-1447, 2010.

30. Leis O, Eguiara A, Lopez-Arribillaga E, et al: Sox 2 expression in breast tumours and activation in breast cancer stem cells. Oncogene 31: 1354-1365, 2012.

31. Dragutinović VV, Radonjić NV, Petronijević ND, et al: Matrix metalloproteinase-2 (MMP-2) and -9 (MMP-9) in preoperative serum as independent prognostic markers in patients with colorectal cancer. Mol Cell Biochem 355: 173-178, 2011.

32. Ispanovic $E$ and Haas TL: JNK and PI3K differentially regulate MMP-2 and MT1-MMP mRNA and protein in response to actin cytoskeleton reorganization in endothelial cells. Am J Physiol Cell Physiol 291: C579-C588, 2006.

33. Bae IH, Park MJ, Yoon SH, et al: Bcl-w promotes gastric cancer cell invasion by inducing matrix metalloproteinase- 2 expression via phosphoinositide 3-kinase, Akt, and Sp1. Cancer Res 66: 4991-4995, 2006. 